\title{
Radiographic Evaluation of Zinc Oxide Eugenol as a Primary Teeth Root Canal Obturation Material on the Permanent Germ Condition (Cases Report and Literature Review)
}

\author{
Dharli Syafriza ${ }^{1},{ }^{*}$ Nova Rosdiana ${ }^{2}$ \\ ${ }^{l}$ Department of Pediatric Dentistry, Faculty of Dentistry, Universitas Syiah Kuala \\ ${ }^{2}$ Department of Dental Radiology, Faculty of Dentistry, Universitas Syiah Kuala \\ *Corresponding author: dharlisyafriza@unsyiah.ac.id
}

\begin{abstract}
Various obturation materials for the root canal of primary teeth are available, but none material that meets as an ideal properties. One of the obturation materials is zinc oxide eugenol (ZOE), with various advantages and disadvantages but is still often used. This case report discussed the radiographic evaluation of several cases of using ZOE as an obturation material for primary teeth against the condition of the permanent tooth germs as well as a review of the literature. All cases showed ZOE resorption rate was slower than root resorption, retained, and as a mechanical barrier against the eruption of permanent teeth. ZOE can be considered as a preferred obturation material for primary teeth, but we should reguler evaluate the permanent germ clinically and radiographically
\end{abstract}

Keywords: zinc oxide eugenol, obturation material, primary teeth, permanent teeth germ

\section{INTRODUCTION}

Several clinicians have noted that the ideal requirements for obturating materials of primary teeth include non-irritating to the periapical tissue, stable desinfected potency, excess material at the apical level that must be absorbed easily, easily inserted into the root canal, easily removed if necessary, adhere to the canal wall, should not shrink and dissolve in water, should not cause tooth discoloration, radiopaque, do not harm the surrounding tooth germ, and do not interfere with the eruption of permanent teeth $[1,2]$.

Zinc oxide eugenol (ZOE) has been used for a long time in dentistry and was the first material recommended for obturation of primary teeth since 1930. Various studies have reported the advantages and disadvantages of $\mathrm{ZOE}$ as a root canal obturation material for primary teeth. ZOE has anti-inflammatory and analgesic properties, a larger zone of bacterial inhibition, easyly available, radiopaque, cost effective, insoluble in tissue fluids, easy to mix, and good working time $[2,3]$.

The success rate of ZOE for obturation of primary teeth reaches $80-96 \%$ which was attributed to its anti- inflammatory and analgesic properties [4] but its presents limited antimicrobial action. Another disadvantage of $\mathrm{ZOE}$ is the slower rate of resorption compared to root resorption, cementum and bone necrosis, so that ZOE cannot be considered as the most ideal root canal filling material.3 Even so, until now ZOE is most often used as an obturation material for primary teeth, including in Indonesia $[5,6]$.

This cases report discussed about the radiographic evaluation and literature review of $\mathrm{ZOE}$ as a root canal obturation material for primary teeth on the condition of permanent tooth germs.

\section{CASES PRESENTATION}

\subsection{Case 1}

A 4.5 year old girl had an exposed pulp fracture of tooth 61 as a result of a fall a week ago. The child has difficulty eating and drinking because it hurts when the tooth touched. The patient had no history of systemic and allergic reactions. Intraoral examination revealed 61 fractures of $1 / 3$ crown with pulp exposed and protruding coronally. After obtaining informed consent, vital pulpectomy was performed on tooth 61 and the root canal 
was obturated with ZOE cement and liner with GIC. Treatment control one week later found no complaints on subjective and objective examination, and continued with the final restoration.

Three years later the patient came back with complaints of loose teeth. A periapical radiograph was taken to see the condition of the 21 germ (Figure 1), it appears that the root of tooth 61 has been resorption up to $1 / 3$ cervical on the mesial side, while on the distal side it has been completely resorption. The radiopaque appearance of the ZOE filler appears to have not been completely adsorbed and appears to remain $\pm 3 \mathrm{~mm}$ from the orifice. Tooth 21 have unerupted, while 11 were fully erupted and incompletely formed root apex. The orientation of the growth of the seed 21 was slightly distal and there was no visible alveolar bone obstructing the eruption. The crown has been fully formed without any abnormalities and root growth has reached the middle $1 / 3$. The recommended treatment for 61 was extraction under infiltration anesthesia because the ZOE material was considered to retain eruption of 61 .

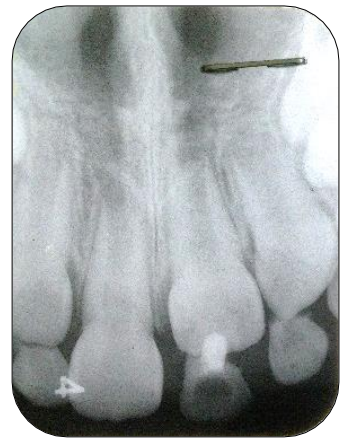

Figure 1. Periapical radiograph of case 1 when the patient was 6.5 years old

\subsection{Case 2}

A 1 year 10 months girl came with complaints of swelling in the gingiva of anterior upper arch, pain history when sleeping at night and had a fever. The patient had no history of systemic and allergic reactions. Intraoral examination showed 51 and 61 caries with nonvital pulp, sensitive to palpation and percussion, mild mobility, redness of the gingiva, and swelling. At the first visit, the pulp chamber was deroofed (access opening), the root canal was cleaned from the necrotic pulp, followed by root canal irrigation, prescribed antibiotics and antipyretics. The second visit (after 5 days) was done by re-cleaning the root canal, determining the working length of root, and administering intrapulpal medicament and restored with a temporary restoration. The patient came back for a third visit (5-7 days later) for root canal obturation with ZOE. Treatment control one week later found no complaints on subjective and objective examination, and continued with the final restoration.
When the patient was 6 years old, she came back with chief complaint of mobility of tooth 51. Periapical radiograph of regions 51 and 61 were taken, showing that the root of tooth 51 had been completely resorption on both the mesial and distal sides. The radiopaque appearance of the ZOE filling material appears to have not been completely absorbed, fractured, and appears to remain $\pm 5.5 \mathrm{~mm}$ from the orifice. The condition of the 11 germ was good with a vertical growth orientation and parallel to the 21 germ. There was no visible alveolar bone that prevented tooth 11 from erupting. The crown has been fully formed without any abnormalities, root growth reaches the middle $1 / 3$. In tooth 61 root resorption reached the middle $1 / 3$. The radiopaque appearance of the ZOE filling material appears to be $\pm 7 \mathrm{~mm}$ remaining from the orifice. The condition of the seedling of tooth 21 was good with a vertical growth orientation. There was no visible alveolar bone that prevented tooth 21 from erupting. The crown has been fully formed without any abnormalities, root growth reaches the middle 1/3.

The treatment for 51 was removed the tooth under infiltration anesthesia due to the fracture of $\mathrm{ZOE}$ material. The remaining fractured $\mathrm{ZOE}$ was removed by curettage carefully. The 61 was remains advised to be observed every 3 months.

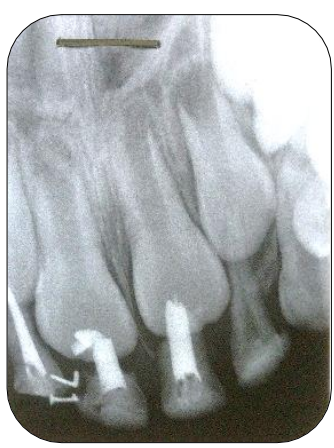

Figure 2. Periapical radiograph of case 2 when the patient was 6 years old

\subsection{Case 3}

A 7.5 year old boy came with a complaint that the restoration which was restored about 1 year ago has detached. Intraoral examination showed that 85 caries with nonvital pulp, negative percussion and palpation, and no redness of the gingiva. The treatment plan was multiple pulpectomy, where at the first visit the pulp deroofed (access opening), root canal cleaning from necrotic pulp and followed by root canal irrigation, determination of working length, and intrapulpal medicament using calcium hydroxide. At the second visit (1 week later), the root canal was obturated using ZOE and lining with a GIC liner. Treatment control one week later found no complaints on subjective and objective examination, and continued with the final restoration. 
When the patient was 10 years old, a panoramic photo was taken and it appeared that the root canal treatment of tooth 85 was still good. The root of tooth 85 underwent minimal resorption. The radiopaque image of the ZOE filler appears intact. The condition of the teeth 45 appears to be good with a slightly distal orientation of growth. The crown has been fully formed without any abnormalities, root growth reaches $1 / 3$ cervical. The condition of the 45 germ did not seem to be in line with the 35 that had erupted and the root formation had reached the middle $1 / 3$.

Based on the radiographic interpretation, the extraction of tooth 85 was postponed and observations were still carried out every 3 months to evaluate the condition of 45 germ.

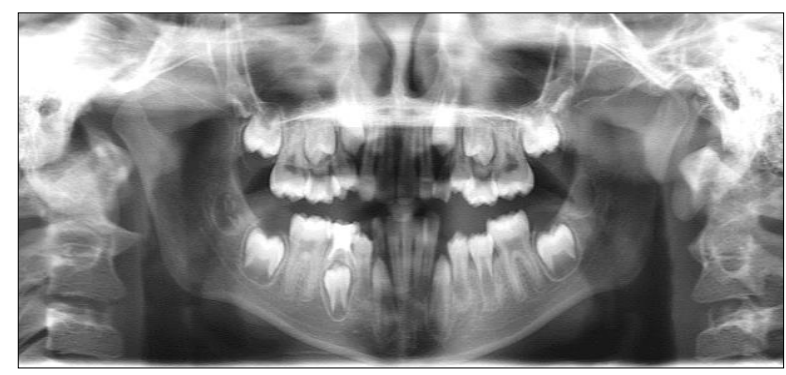

Figure 3. Panoramic radiograph of case 3 when the patient was 10 years old

\subsection{Case 4}

A 5 year old boy came with a complaint of cavities in the left lower jaw. Intraoral examination revealed that 75 caries, nonvital pulp, negative percussion and palpation, and no redness of the gingiva. At the first visit, the pulpectomy treatment was starting with pulp deroofed (access opening), cleaning the root canal from necrotic pulp followed by root canal irrigation, determining the working length, and intrapulpal medicament using calcium hydroxide. One week later the patient came back for a second visit. There were no complaints during subjective and objective examination, and continued with obturation of the root canal using ZOE and lining with a GIC liner. Treatment control one week later found no complaints on subjective and objective examination, and continued with the final restoration.

When the patient was 7.5 years old, radiographic examination was performed. The root canal treatment of tooth 75 was in good condition where the radiopaque appearance of the ZOE filling material appeared intact at the mesial root but the distal root appeared fractured due to pressure from the underlying permanent tooth 35 . The condition of 35 germ good with a vertical growth orientation. Root growth reaches $1 / 3$ cervical. It was seen that tooth 75 was not supported by alveolar bone on the distal side and the crown area of tooth 35 was radiolucent and extended to the furcation. With this interpretation, it was decided that the treatment plan for tooth 75 was extraction with infiltration anesthesia.

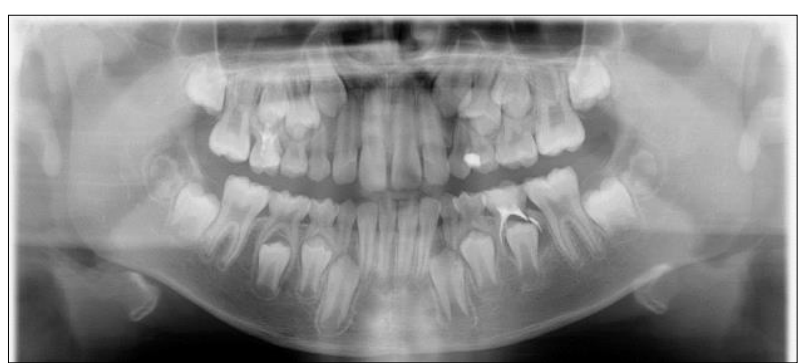

Figure 4. Panoramic radiograph of case 4 when the patient was 7.5 years old

\section{DISCUSSION \\ AND \\ REVIEW \\ LITERATURE}

Primary teeth with root canals infection which has reached periapical tissue are common that can cause premature loss of primary teeth if left untreated. It is important to maintained the primary teeth, remain free from the infected disease as a natural space maintainer. Root canal treatment is indicated for primary teeth with irreversible or necrotic pulp in order to maintain them in a functional state until the permanent teeth erupt. Successful root canal treatment supported by obturation materials with antimicrobial properties.

Zinc oxide eugenol (ZOE) has been used as a root canal obturation material for primary teeth started in 1930 and made ZOE as one of the oldest root canal materials for primary teeth compared to other materials [3]. The American Academy of Pediatric Dentistry (AAPD) in 2008 explicitly recommended only ZOE as the material of choice for primary teeth root canal obturation [7].

Success rate of ZOE as obturation material for primary teeth in several study reported to varied widely. This success rates variation was due to differences in treatment protocols, inclusion criteria of treated teeth, timing of control, different clinical and radiographic definitions, or both. A retrospective study in Iran (2015) showed that two visit pulpectomy with ZOE as root canal filling material was successful in $93.4 \%$ of the teeth treated as verified by clinical and radiographic examinations [8]. Clinical and radiographic success rates of ZOE in other study was $100 \%$ at 6 and 12 months. None molar treated failed at 6 and 12 months, but after 18 months evaluation showed radiologically failed from six molars [9]. Another study in 2011 showed the clinical and radiographic success rates of ZOE was $85.71 \%$ after a period of 6 months. Thus, ZOE as an obturation material has been shown to reduce clinical signs and symptoms and cause healing of periapical pathologies [10].

The success rate of $\mathrm{ZOE}$ as an obturation material for primary teeth is due to the properties and advantages of 
ZOE including anti-inflammatory and analgesic, the greater zone of bacterial inhibition, ease of availability, radiopacity of material, cheaper/cost effective, good plasticity, insolubility in tissue fluids, easy to mix and good working time [2]. The amount of eugenol released in the periapical zone immediately after placement was 10-4 and falls to 10-6 after 24 hours. Within these concentrations, eugenol is said to have an antiinflammatory and analgesic properties [11]. In fact, the biological effects of eugenol vary greatly, depending on its concentration. Eugenol may have a beneficial effect, with concentrations ranging from $10^{-8}$ to $10^{-5}$ (prostaglandin synthesis, nerve activity, and white blood cells chemotaxis inhibition), but may be cytotoxic with concentration $>10^{-3}$ (cell death, cell growth, and respiration inhibition) [12].

Although the success rate of $\mathrm{ZOE}$ as an obturation material for primary teeth is quite high, it cannot be considered as an ideal root canal filling material because as it presents limited antimicrobial action and slower rate than primary root resorption. Basically, formation of ZOE results from the reaction of eugenol and bivalent zinc ions to form insoluble chelation, wrapping remanent zinc oxide in it and forming a solid mass [9]. Because $\mathrm{ZOE}$ is a solid mass, its resorbability is more slowly and uneasily to dissolves. A research concluded, that retained ZOE was not related to pulpectomy success or failure, pulpectomy preoperative, root resorption, age of the patient at time of treatment, whether the pulpectomized tooth was extracted or exfoliated, or the timing of the pulpectomy's loss [3]. All cases in this report show a fairly good success rate of pulpectomy treatments. Radiographically, ZOE resorption was slower than root resorption. Cases 1 and 2 showed that almost all of the roots had been resorption while the $\mathrm{ZOE}$ remained in the root canal. Roots resorption in all cases was a physiological process that occurs in primary teeth that characterized by the activity of clastic cells resulting in loss of cementum and dentin in the root area. As there was a difference between the resorption rate of the zinc oxide paste and the tooth, radiographic checks should be made regularly at intervals of 1-2 years [7].

When ZOE extrudes dan its excess forced through the apex, its can develops a fibrous capsule which prevents resorption of $\mathrm{ZOE}$ and tends to be retained in the apical tissue because ZOE takes few months or even years to resorb [14]. Previous research concludes that two-thirds extruded ZOE group was not resorbed at 12 months and about one-thirds at 18 months [9]. Another research indicated that retained $\mathrm{ZOE}$ tend to resorb with time and may reflect the osteoclast activity to reduce $\mathrm{ZOE}$ particles. The mean time for ZOE to resorb about 50.1 months, $80 \%$ showed significant reduction of size by 5 $\mathrm{mm}$ or more over time. The differences of reduction time findings may reflect differences ability to resorb between species [13].
This extruded and retained ZOE was not related to both clinical and radiographic success rate, but can caused permanent tooth germ to erupt abnormally. In case 1 and 3 showed delayed eruption of the permanent teeth when compared to adjacent or opposite teeth. It appears that eruption of 21 (case 1) and 45 (case 3 ) was retained by the presence of $\mathrm{ZOE}$ in the unresorbed root canal. This might be related to ZOE formation as a solid mass and uneasily to dissolves that forms a mechanical barrier against the eruption of permanent teeth. As reported, ZOE resisted resorption and might deflect the path of eruption of the succedaneous tooth in about $20 \%$ cases. There were $4.1 \%(3 / 74)$ tooth's lost about 6 or more months later than normal, and $29.6 \%$ (24/81) were extracted because they were loose but over retained when the permanent tooth was erupting. Other conditions that can be found were incidence of anterior cross bites or palatal eruption of permanent incisor $(20 \%)$, and over retention with ectopic eruption of the succedaneous molar $(21.6 \%)$ [15]

In contrast to cases 2 and 4 , the position of the permanent tooth germ was at the same level but the ZOE at the apex was fractured and separated from the ZOE in the root canal. The fractured ZOE caused no apparent pathology radiographically or clinical examination. This is indicated that ZOE particles cause no harm even not resorbed. It is possible to remove retained $\mathrm{ZOE}$ by carefully curettage immediately after exfoliation or extraction [13].

Authors suggested and seem advisable a short-filled pulpectomies (1 mm or more short of the apex) than fills beyond the root or those filled completely to the apex to ovoid retained ZOE. Overfilling is not preferable because of the side effects of zinc oxide-eugenol beyond the root apex that initiation a foreign body reaction. Once ZOE forms a hardened state, its resorption may be delayed and resulted a succedaneous tooth deflection $[15,16]$.

\section{CONCLUSION}

Zinc Oxide Eugenol (ZOE) still possible to be used as a root canal obturation material for primary teeth, but in its use it is strongly recommended to carry out follow-up and regular observations of the condition of the permanent teeth to avoid various effects.

\section{REFERENCES}

[1] Holan G, Fuks AB. A comparison of pulpectomies using ZOE and KRI paste in primary molars: a retrospective study. Pediatr Dent 15:403-407, 1993

[2] Chonat A, Rajamani T, Ephralm R. Obturating Materials in Primary Teeth-A Review. RRJDS 6: Issue 1,2018 
[3] Gupta B, Singh I, Goyal P, Garg S, Gupta S. A Clinical and Radiographic Study of Four Different Root Canal Filling Materials in Primary Molars-An In Vivo Study. Dent J Adv Stud 7: 61-65, 2019

[4] Marwah N. Textbook of Pediatric Dentistry. 3rd Ed. Jaypee Brothers Medical. Publishers (P) Ltd.; 2014. p. 672

[5] Damayanti A, Kaswindiarti S. Perawatan Pulpektomi Non Vital Pada Gigi Desidui Anterior Maksila (Laporan Kasus). JIKG 1: 58-63, 2017

[6] Pediarahma A, Rizal MF. Zinc Oxide EugenolFormocresol Root Canal Treatment Fails to Treat a Deciduous Tooth with Dentoalveolar Abcess. Case Report. J Dent Ind 3:100-104, 2014

[7] American Academy of Pediatric Dentistry. Pulp therapy for primary and immature permanent teeth. The Reference Manual of Pediatric Dentistry. Chicago, Ill.: American Academy of Pediatric Dentistry: 384-92, 2020

[8] Bahrololoomi Z, Zamaninejad S. Success Rate of Zinc Oxide Eugenol in Pulpectomy of Necrotic Primary Molars: A Retrospective Study. JDMT 2: 89-94, 2015

[9] Chen X, Liu X, Zhong J. Clinical and Radiographic Evaluation Of Pulpectomy In Primary Teeth: A 18Months Clinical Randomized Controlled Trial. Head \& Face Medicine 13:12, 2017

[10] Gupta S, Das G. Clinical and radiographic evaluation of zinc oxide eugenol and metapex in root canal treatment of primary teeth. J Indian Soc Pedod Prev Dent 29:222-8, 2011

[11] Hashieh IA, Pommel L, Camps J. Concentration of Eugenol Apically Released from Zinc OxideEugenoI-Based Sealers. J Endodon 11: 713-15, 1999

[12] Markowitz K, Moynihan M, Liu M, Kim S. Biological properties of eugenol and zinc oxideeugenol. Oral Surg Oral Med Oral Pathol 73: 72937, 1992

[13] Sadrian R, Coil JA. A Long-Term Followup on the Retention Rate of Zinc Oxide Eugenol Filler after Primary Tooth Pulpectomy. Ped Dent 4: 249-53, 1993

[14] Kannan R, Mathew MG. Pediatric obturating materials - A review. Drug Invention Today 11: Issue 1, 2019

[15] Coll JA, Sadrian R. Predicting pulpectomy success and its relationship to exfoliation and succedaneous dentition. Pediatr Dent 18:57-63, 1996
[16] Yacobi R, Kenny DJ, Johnston DH. Evolving primary pulp therapy techniques. J Am Dent Assoc122 (2):83-5 1991 